\title{
Classroom features supporting free-choice transfer
}

\author{
Leslie Atkins Elliott \\ Curriculum, Instruction and Foundational Studies, Boise State University, \\ 1910 University Drive, ED-217, Boise, ID 83725-1747
}

\begin{abstract}
This paper reports on characteristics of a class that consistently supports free-choice transfer - transfer "where the application of prior learning is possible but is not required" (Pugh, 2011). We review literature that hypothesizes instructional supports for such transfer - TTES, accountable authoring, expansive framing, and expansive learning - and demonstrate how these are, or are not, instantiated in this physics course.
\end{abstract}

\section{INTRODUCTION}

Our group is working on the idea of how glasses and contacts change the shape of your cornea... [We thought] people with far sighted vision need glasses with thicker glass in the center... When I was at Walgreen's the other day, I saw some reading glasses and decided to investigate. And sure enough, the glasses were thicker in the center and as the intensity of the prescription increased, so did the thickness... I was so proud of our group to turn out correct! -Maddy

Physics Education Research has made great strides in improving students' understanding of physics content by operationalizing "understanding" [1], creating curricula that promote gains in understanding, and developing common design principles for that curriculum [2]. However, this improved understanding is of little value if students do not draw on their knowledge in non-instructional contexts. And yet there is little research on whether or not students transfer ideas from physics classes to those settings, nor does PER attend specifically to design principles that promote that transfer.

This gap is the rationale behind the study reported here. Specifically, we have been interested in one type of transfer: moments in which students use science concepts to see and experience their "everyday" world in meaningful, new waysa concept related to Pugh's [3] construct of "transformative experience" (TE). In other papers we examine the measurement of TE $[4,5]$. In this paper we address the question: what aspects of curriculum and instruction support high TE?

Below, we summarize research on characteristics of instruction that promote or inhibit transfer [6-9]. We then examine how those characteristics are instantiated in a physics classroom that has high TE scores [5]. Although we are reporting on a non-traditional course, we anticipate that these findings can lead to design principles to support transfer in more traditional courses.

\section{BACKGROUND: LEARNING FOR TRANSFER}

The majority of research on transfer has been conducted with scripted training and defined target scenarios in pscyhology labs; findings emphasize the content knowledge and similarity between training and target contexts [10]. In our study, we are interested in learning that takes place in the less-controlled setting of a classroom, and transfer as a choice to notice and use ideas from class in settings where it is not required or even anticipated. Despite the large literature on transfer, studies of this ilk - particularly in science - are few; we briefly summarize four strands of research below, connecting these with the introductory quote by Maddy (an example of a transformative experience), before using these four studies as a lens to examime classroom activity.

\section{A. Pugh: teaching for TE in science (TTES)}

To teach for TE, Pugh notes that instructors should "create a context where particular ways of experiencing the world through concepts are displayed and valued and to help students come to participate more centrally in these experiences." (p 1106) To do this, a teacher should "(a) [frame] the content in terms of its experiential value, (b) [scaffold] re-seeing, and (c) [model] transformative experiences."

This approach foregrounds particular ways an instructor positions the content: noting for students how content has use and value, modeling how to see the world with these concepts, and scaffolding students in seeing the world in new ways. This stands in contrast to approaches described below, which attend to how classroom activity (particularly the construction of content), rather than the content itself, is framed.

Maddy's experience at Walgreen's, in Pugh's model, would emerge from specific framing by the instructor (e.g., "learning about lenses can help you to understand your own vision problems"), modeling by the instructor (e.g., "I used our ideas about focus when I saw..."), and scaffolds. In section IV, we will examine how these are, or are not, at play in instruction.

\section{B. Greeno: accountable authors}

In his preface to a journal issue on transfer, Greeno [8] hypothesizes that transfer happens, in part, when "...people learn how to act with conceptual agency in substantive domains and in activity settings, and authoritative and accountable positioning in learning environments facilitates that learning." He adds, "If a student has developed a participatory identity with strong conceptual agency while learning concepts...we could 
expect that student to be more likely to participate with strong conceptual agency ... in another setting."

In Greeno's description, when Maddy uses reading glasses to confirm physics ideas, she must not only understand reflection and refraction, but have the conceptual agency to co-opt physics ideas to interpret novel scenarios. She treats ideas and methods as "resources that can be adapted, evaluated, questioned, and modified." Agency, he proposes, is facilitated by positioning Maddy as an accountable author of ideas. This departs from traditional models of transfer, which have not attended to students' agency with content. This contrasts, too, with Pugh's approach, which may encourage agency but does not explicitly position students as authors of scientific concepts as a means to achieving agency.

\section{Engle: expansive framing}

Engle[7] also examines how students and content are positioned in a classroom that shows evidence of free-choice transfer. Her ideas echo Greeno, with an emphasis on accountable authorship, as well as Pugh, as she highlights the role of an instructor in explicitly noting that students should "continue using what they have learned." In addition, and drawing on research that shows similarities between "training" and "target" contexts facilitate transfer, she argues for classes that expansively frame the learning context so that "a larger encompassing context is formed that seamlessly incorporates learning and transfer contexts."

In this description, investigating glasses at Walgreens is indicative of expansive framing: Walgreens becomes part of our class activity - essentially serving as Maddy's lab, where implications of ideas developed in class are vetted, rather than a separate context that is "outside" of class. In section IV, we will examine how the class achieves expansive framing.

\section{Engeström: overcoming encapsulation}

Finally, we turn to research that examines the opposite of transfer: "encapsulation," where ideas have use and relevance in a narrow context. Engeström[9] highlights one example: students' explanations of the phases of the moon, even after instruction, are disconnected from any observed properties of the moon. These misconceptions, he argues, "are not indications of immature thinking. They are culturally produced artifacts..." In his Activity Theory analysis, he suggests that school activity is such that "the school text is the object of the activity instead of being an instrument for understanding the world." Students learn about the textbook, rather than using the textbook to learn about the moon. A range of characteristics of curriculum and instruction - not just textbooks - serve to encapsulate learning.

Engeström argues that to construct classrooms that are not encapsualted, one must shift towards expansive learning, where "learners construct a new object and concept for their collective activity, and implement this new object and concept in practice... Nobody knows exactly what needs to be learned. The design of the new activity and the acquisition of the knowledge and skills it requires are increasingly intertwined" (1999).

These implications are reminiscent of Greeno (students as accountable authors of content) and of Engle (instead of a bounded context/text, there is an open - e.g., expansively framed - 'context of discovery'"). But for Engeström this is relevant not only because it provides students with conceptual agency and reduces barriers for transfer, but because it will demand that their inquiry is not a question or object particular to school, but discovered by and relevant to students.

For Maddy, the question of lens shape is an activity that her group has constructed. The methods of discovery were their own (they drew on lenses with markers to trace the path of light), and were related to their representation of focus. Seeing reading glasses at Walgreens as an opportunity to confirm physics ideas emerges from this "expansive learning." We can imagine that, had class been structured to cover the lens-makers equation, using Pacso equipment (materials that suggest encapsulation and not expansive framing), she would be unlikely to see reading glasses at Walgreens as an opportunity to confirm those ideas, and doing so would be less likely to be a source of great pride. In section IV we will see how the class supports those inquiries and expansive learning.

\section{DATA: SCIENTIFIC INQUIRY CLASS}

We have developed and implemented a survey on transformative experiences [4] and identified a course with high TE: Scientific Inquiry. Briefly, this is a course for undergraduate pre-service elementary teachers; we have no textbook, lab manual or typical lab materials, but work in small groups with everyday materials to model complex phenomena. We have frequent whole-class conversations as we develop consensus models $[11,12]$. The class is videotaped and the instructor maintains field notes. The course was designed to engage students in inquiry, not transfer, nor was it based on the principles (described above) that promote transfer and TE. Instead, we choose content that is easy to investigate and model, connected to everyday experiences, and easy to make progress in, but also a topic with "depth."

Because the course has high TE scores, we examine this course to see whether and how those principles are instantiated. For this paper, we have transcribed a relatively typical day: students began the semester by examining a pinhole camera and constructing models to explain it; they have nascent models for how light enters the pinhole to produce an inverted image. Students have recently questioned how the light reflects off of surfaces so that it enters the pinhole, and several groups have been experimenting to answer that question. The day begins with the instructor (the author) summarizing the class model, the problem that model raised (how light reflects), current ideas that have been proposed to solve 


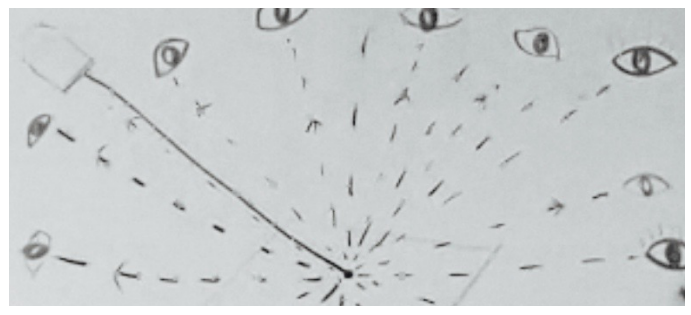

FIG. 1. Diagram of laser light fragmenting off paper.

that problem, and then asking for additional ideas.

\section{SCIENTIFIC INQUIRY: SUPPORTING TRANSFER}

In this section, we trace activity of one 100-minute class in light of the ideas of TTES, accountable authorship, expansive framing, and overcoming encapsulation. Above we presented those ideas in order from content, to authorship of content, to the framing of activity, and then the broader description of expansive learning. Below, we reverse this order, starting with the nature of learning in this class (expansive learning), then how that learning is framed (expansive framing), how content is constructed (accountable authorship) and, finally, how the teacher positions content (TTES).

\section{A. Overcoming encapsulation: expansive learning}

When soliciting models for how light reflects from surfaces to enter the pinhole, one group offers the following: "it [the incoming ray] kind of breaks into lots of rays that go out in all directions." This idea (from Marie) explains how light is reflected into the pinhole camera, but another student, Darcy, argues that her group's data refutes this model. They used chalk dust, a laser pointer and paper. Though they could see a ray coming to the paper, they saw no rays reflecting back: "If it were to fragment, we'd be able to see it. Even if it was dim and faint, we'd be able to see the visual rays in the dust. And we didn't." We work to clarify the model, with Sarah noting: "I don't think they're saying there's five [rays] coming off [as shown on their diagram]. It's infinity coming off...there's so many you wouldn't be able to see those rays." Once we clarify this, recreating Darcy's group's experiment for the class, and Marie's group creates Figure 1. Alyssa is not convinced. She notes that this model suggests that objects, like a desk, should appear to glow:

Alyssa: Wouldn't we be seeing it as a shiny brown, like the [brown] desk would look like it was kind of lit up. Like in the cartoons where they get an idea and the light bulb's kind of like fuzzy? So wouldn't the desk be all fuzzy brown...you know what I mean?

Instructor (Leslie): It would be glowing.

Alyssa: Exactly. It'd be glowing. And nothing's glowing when you look at it.
Though a full activity-theory analysis is beyond the scope of this paper, we see several indications that students are engaging in expansive learning: without a textbook to serve as the object of inquiry, students are working to understand phenomena and each others' ideas. Without traditional lab materials, they are improvising with everyday materials: a laser pointer, paper, chalkdust, bathroom mirrors. They have constructed a concept and object for their inquiry: what does it mean for something to glow? and how does our model of fragmentation (what a physicist would call diffuse reflection) differ from models of glowing?

Note that the model that Darcy objects to is essentially what is in many textbooks: rays reflecting off in multiple directions as a representation of diffuse reflection. The distinction lies not in the substance of the content, but the approach.

\section{B. Reducing barriers: expansive framing}

In addition to engaging in expansive learning, we can see evidence in class that students are expansively framing their activity. They do not refer only to in-class objects and questions, but they draw on both in- and out-of-class contexts. When discussing the reflection of laser light, Marie notes it is "more like a car headlight when it's foggy out. You just see...a lit-up area rather than a singular ray." In the transcript above, Darcy uses a wooden desk as a representative "nonshiny" object. She explains her idea in terms of the glow of a cartoon lightbulb. Later, as students contemplate whether or not objects are glowing, Melissa describes someone under a spotlight, and Marie relates this to videotapes from dance performances: "When the stage is all dark, and there's a spotlight on one person, that... person has a fuzzy glow around them...it's the same idea as the Moon and space, when everything around you is dark and you're the only thing fragmenting that light, I feel like it has that like 'aaaaah' around you."

In these examples, we see that the explanations they generate in class are relevant not only to the very particular classroom objects, but it is framed more broadly: modeling a pinhole camera draws on evidence from dance performances and moonlight. Engle suggests such framing promotes transfer by making transfer contexts part of the learning context. We can imagine that, as Engle hypothesizes, these students are more likely to use ideas of diffuse reflection when outside of class, because those contexts have been employed as they develop and vet ideas.

Another form of expansive framing [14] is "temporally connecting to prior and future interactions" linking prior temporal contexts to the current context. The course is continually building on and refining prior ideas and models. We begin the day by looking back at open questions. Homework generally draws on the ideas that have emerged from the week, and students' responses to homework become the material for the next week's class. 


\section{Conceptual agency: accountable authors}

Greeno posits that transfer is facilitated when students have conceptual agency, and such agency is developed by positioning students as accountable authors of content. The class is structured to require students to author ideas: without lectures or books, they are responsible for constructing the content of the class. In addition, a central aspect of the course is ongoing peer feedback as they share, vet, critique and refine ideas. That is, Marie's model must address Darcy's evidence and Alyssa's concerns. And Alyssa's ideas about what is and is not glowing is accountable to Marie's experience of spotlights.

Authorship is not only supported through the lack of text or lecture, but through explicit support by the instructor. I begin the day by revisiting models that have been developed and noting the authors of those models: "this group's idea..." (that light rays bounce around until they finally enter the pinhole) and "Balyn's model" (that surfaces are so bumpy that light can simply reflect right into the pinhole). Indeed, naming models after authors is a common practice in this class.

Accountability emerges not only from students' questions to one another, but through a range of pedagogical moves that hold students accountable to other students' critiques and disciplinary norms. In this class, we share whiteboards that model fragmentation and discrepancies in those models must be addressed. Homework generally builds on students' ideas and is subject to peer review[12]. Agency, then, is promoted not only by authoring ideas but by continually subjecting those ideas to scrutiny - evaluated, adapted and modified as a part of that authorship.

\section{Teaching for TE: modeling and scaffolding}

When reviewing the 100-minute class for evidence that the instructor employs elements of the TTES model (that is, model TE, describe experiential value, or explicitly scaffold "re-seeing"), such instances are rare. The only explicit instance occurs when I offer an example of glowing as we consider Alyssa's challenge: "I thought of the Moon. The Moon is not lit from within, but it looks like it's glowing."
We attribute this, in part, to the following: When we expansively learn, the questions are motivated by students' emerging interests, so telling students why the ideas have experiential value is superfluous; they are valuable because the ideas answer their questions and resolve problems they find vexing. When we expansively frame activity, the instructor does not need to model TE or scaffold re-seeing: other contexts become part of instruction, and are often brought in by the students. Scaffolds for TE, then, need not be explicit - we can design courses that cultivate students' interest, encourage them to draw on out-of-school contexts, and support re-seeing without explicitly demonstrating or modeling these activities.

\section{CONCLUSION: CHALLENGES, \& DIRECTIONS}

Teaching for transfer has long been a goal of instruction, but remains elusive in most studies. In this paper, we examine a course that demonstrates transfer of a particular type - freechoice transfer in which students notice, value and use ideas from physics (TE) - and examine how methods to support transfer are instantiated in the course. We find that expansive learning, expansive framing, and accountable authorship feature prominently in class, and are likely to support TE. Furthermore, these approaches appear to mitigate a need for more explicit scaffolding of TE.

However, the course described here is not traditional - we discuss three topics in depth (light, color and the eye), and the particular representations and content constructed, while sharing commonalities from one semester to the next, also has more variation than in traditional courses. That is to say, employing these design principles in more typical PER-based instruction is not trivial. Nonetheless, we can imagine instruction in PER-based courses that draws on these findings and promotes expansive framing, accountable authorship and expansive learning.

\section{ACKNOWLEDGMENTS}

Thank you to Angela Little for feedback and input on this article. Research supported by NSF \# 1140785.
[1] A Madsen, SB McKagan, \& EC Sayre. arXiv:1605.02703 (2016).

[2] DE Meltzer \& RK Thornton, Am. J. Phys. 80, 478 (2012).

[3] KJ Pugh, L Linnenbrink-Garcia, KL Koskey, VC Stewart,\& C Manzey. Sci. Ed. 94, 1-28 (2010).

[4] B Frank, \& P Mittura, PERC Proceedings (2014).

[5] B Frank \& LJ Atkins, PERC Proceedings (2013).

[6] KJ Pugh, L Linnenbrink-Garcia, KL Koskey, VC Stewart,\& C Manzey. Cogn. \& Inst. 28, 273 (2010).

[7] RA Engle. J. Learn. Sci. 15, 451 (2006).

[8] JG Greeno. J. Learn. Sci. 15, 537 (2006).
[9] Y Engestrom, Learn. \& Inst. 1 243(1991).

[10] SM Barnett \& SJ Ceci. Psych. bulletin 128, 612 (2002).

[11] LJ Atkins \& IY Salter. Chapter in Effective Practices in Preservice Physics Teacher Education, edited by E. Brewe and C. Sandifer. 2015, APS.

[12] L Atkins Elliott, K Jaxon, \& I Salter. Composing Science. NY: Teachers College Press. (2016).

[13] JF Wagner. Cog. \& Inst. 24, 1 (2006).

[14] RA Engle, DP Lam, XS Meyer, \& SE Nix, Ed. Psychologist, 47, 215-231 (2012). 